www.jmscr.igmpublication.org

Index Copernicus Value: 79.54

ISSN (e)-2347-176x ISSN (p) 2455-0450

crossref DOI: https://dx.doi.org/10.18535/jmscr/v7i4.25

\title{
Results of Triceps Aponeurosis Tongue Approach for Distal Humerus Fractures
}

\author{
Authors \\ Dr Bias Dev ${ }^{1}$, Dr Simriti ${ }^{2} *$ \\ ${ }^{1}$ Department of Orthopaedics, GMC Jammu, J\&K, India \\ ${ }^{2}$ B2 Medical Enclave GMC Residential Complex Jammu, J\&K, India \\ Mob: 9419787722,01912578636
}

\begin{abstract}
The retrospective study has been conducted to evaluate the results of intracondylar fractures of distal humerus dealt through posterior Triceps Apponeurosis Tongue Approach ( TATA approach) without necessitating the osteotomy of olecronon in 48 patients from Jan 2010 to Dec 2017. The patients has been followed up for at least one year. The study shows good to excellent results in results in $87 \%$ patients as per MEPI score, while obviating the complication of osteotomy and its fixation.

Keywords: Intercondylar humerus fracture,Triceps aponeurosis tongue approach.
\end{abstract}

\section{Introduction}

The intraarticular fractures of distal humerus are approximately $5 \%$ of total adult fractures and can result in serious disability if not managed adequately. Being intrarticular, need to have open reduction and internal fixation of these fractures is undisputable but the choice of approach, implant and its placement is constantly under peer review $(1,2,3)$. The intrarticular fixation requires visualisation of distal articular surface and stabilisation of both the columns which are best attained through a posterior $\operatorname{approach}^{(1,4)}$. The door to distal humerus opens from the back ${ }^{(1,2)}$. The commonly used approachs are Posterior Campbells approach, posterior approach through olecronon soteotomy, Triceps reflecting anconeus pedicle approach, with each one having its share of merits and demerits ${ }^{(5,6,7)}$.

This study was carried out to analyze the outcomes of fixation of Intraarticular distal humerus fractures through Campbells Triceps Apponeurosis Tongue Approach (TATA approach)

\section{Material \& Methods}

We have included all the intraarticular humerus fractures fixed Using TATA approach from January 2010 onwards till Dec 2017. In this study All the procedures were carried out by the single surgeon . After performing 10 fixations the author performed the cadaveric dissect on for TATA approach in 2011 on five cadaveric specimens. Length, breadth of triceps aponeurosis, intrarticular branch of ulnar nerve and muscle needing detachment for clearing the posterior condyles for the implant placement were visualised for better understanding of the approach. 


\section{Surgical Technique}

Lateral position, regional or general anaesthesia, no tourniquet, arm hanging by side in such a way that on need it could be flexed completely. Posterior midline incision from middle arm 5-7 $\mathrm{cm}$ distal to tip of olecronon curved away from its tip. Ulnar nerve is identified, released proximally and distally and kept away from harms way by putting a tape around it. A rectangular flap of a portion of muscle along with aponeurosis is elevated from proximally to distally upto olecronon leaving a rim of aponeurosis behind for future reattachment. Underneath triceps muscle is split and retracted medially as well as laterally. The elbow is gently flexed, while the olecronon is pulled thrugh aponeurotic tongue. Depending upon the need of fracture fixation, muscle attachments from medial and lateral posterior condyles are erased.

\section{Fracture fixation}

The trochlear component is reduced and held with a $\mathrm{K}$ wire or fixed with partially threaded $4 \mathrm{~mm}$ compression screw followed by reduction of both the columns which are then held temporarily with $\mathrm{K}$ wires. Temporary fixation is followed with application of lateral and medial plate. While fracture fixation, it was ensured that the olecronon process do not get impinged or te plate placement don ot obstruct the olecronon movement. After the procedure, the elbow joint was moved to look for stability and any abnormal sound from the joint while moving it. After suturing both limbs of triceps muscles, Triceps aponeuross was reattached to the rim of aponeurosis. The negative suction was placed in all patients which was removed after 48 hours. After closer of wound and applying dressing, the upper limb was kept in the flexible splnt (Crammer wire). Patient was encouraged to move the elbow while in flexible splint. After suture removal, the splint was removed three times a day for passive, passive assisted, active assisted and active movements with progression of healing. For initial three months or till there were signs $f$ radiological healing patient were called for follow every fortnight.

After radiological signs of union, patient was followed up every three months for one year.

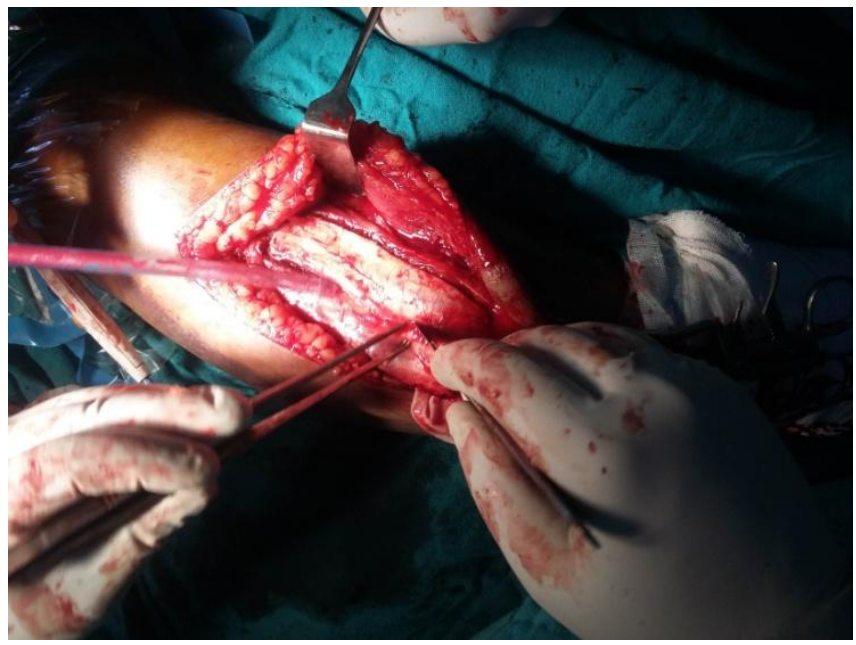

Fig 1

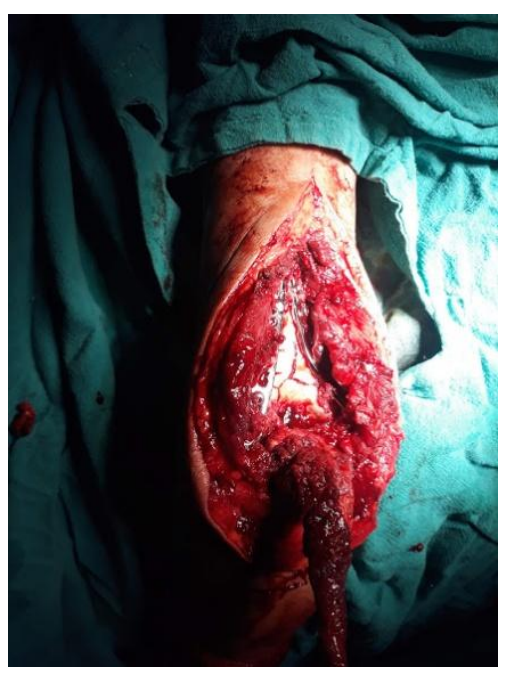

Fig 2

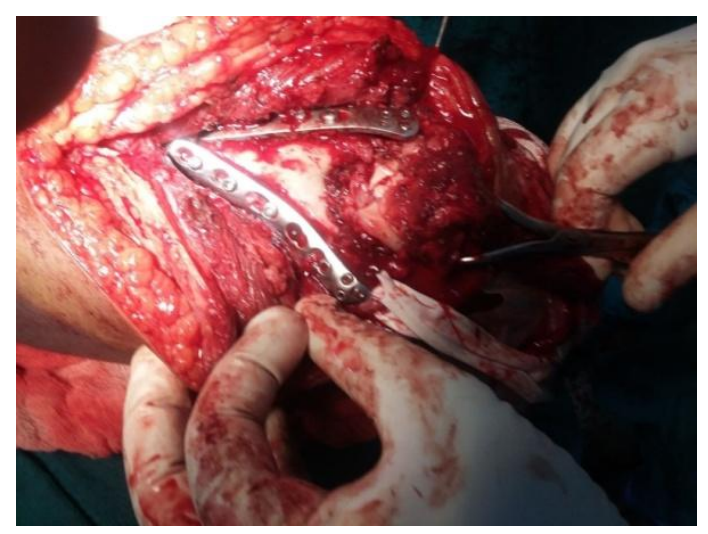

Fig 3 


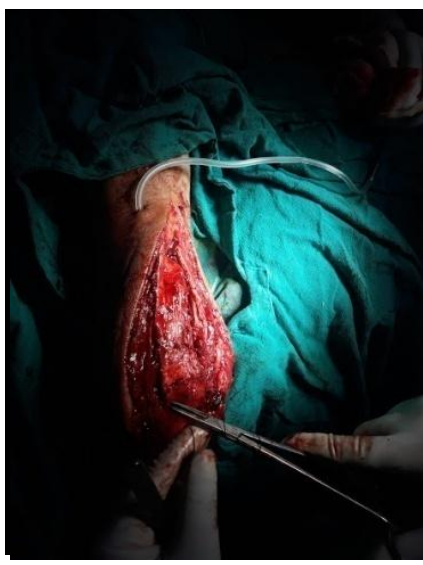

Fig 4

Fig 1, 2,3 \& 4 : surgical steps of creating triceps aponeeurotic tongue, fixation of bone and closure

\section{Results}

There were total 48 patients who were operated with TATA approach from January 2010 to Dec 2017. The patients with follow up of at least one year were 40 . Out of 40 patients 24 were females with average age of 54 years and 16 males with the average age of 42 years.

The surgery was delayed with an average delay of 5 days. There were 6 patients with Type 1 compound fractures, which were operated with in 24 hour of the injury.

Time consumed for surgery was on average 3 hours 40 minutes in initial 10 cases. After exposure to cadaveric dissection the surgical time in next 15 cases was reduced from 3 hours 40 minutes to 3 hours and time was further reduced to 2 hours 45 minutes in rest of the cases.

The blood loss on average has been $220 \mathrm{ml}$, and on no occasion there occured the need for blood transfusion.

There has been no instance of infection in these cases. There were three cases in which there has been marginal skin necrosis. All these cases of skin necrosis settled without needing any subsequent procedure.

All the patients followed up had radiological union by on average 15 weeks. We do not have any patient in this series with non-union.

The average range of extension flexion arc was 5 degree to 110 degrees, with supination pronation being 160 degree. There has been 10 patients who flexion extension arc has been between 50 degree to 60 degree and supination pronation arc as 90 degree. There has been 20 patients whose flexion extension arc is average 100 degree with average supination pronation of 150 degree. In 10 patients the average arc of flexion extension is 120 degree with average flexion extension being comparable to opposite uninjured limb.

Using Mayo elbow performance index, a scale consisting of pain, range of movement, stability and strength of elbow, on the scale of $100,27 \mathrm{had}$ score between 80 and 90 and is graded good, 8 patients had score above 90 and is graded excellent , 3 patients score has been between 70 to 80 and has been graded fair , 2 patients score has been less than 60 and is graded poor.

\section{Discussion}

Elbow being the articulation of ulno humeral, radio ulnar, and radio capitallar joints, any incongruity at any of the joints can cause pain and limit motion, Jeoparadising the functional outcome in distal humerus intrarticular fractures. The direct muscle injury with resultant fibrosis is attributed to cause elbow stiffness. The functional results depend upon good intraarticular reduction, stable fixation and early range of movement exercises $^{(1,2,4)}$. All these desired objectives can be achieved by the TATA approach. $87 \%$ of our patients has shown good to excellent results which has been operated through this approach. In all the cases there was no obstacle to access through this approach.

The posterior exposure of distal humerus through olecronon osteotomy is preferred choice for many but has been associated with higher rates of complications associated with the osteotomy site or fixation method like delayed union, non union, hardware symptoms as reported by various authors $^{(5,6,7)}$. Distal humeral fracture which need fixation of both the columns sometimes do not provide space for stuffing more implant in its vicinity and can cause skin complications ${ }^{(8,9,10)}$. The osteotomy fixation implant often necessitates its remova without inviting any complication of 
osteotomy $^{(11,12)}$. The TATA approach once learned well can deliver good results as has been in our study. The operative time was reduced by 40 minutes after having exposure to anatomy of the region learnt through cadaveric dissection.

\section{Conclusion}

Intrarticular distal humerus fractures which are notorious for its complication in form of stiff elbows can be fixed adequately through TATA approach without inviting complications of osteotomy with resultant stable, painfree elbows with good range of movement.

\section{Bibliography}

1. Pankaj a , Mallinth G, Malhotra R, Bhan $\mathrm{S}$. surgical management of intercondylar fracturs of the humans uing triceps reflecting anconeus pedicle approach. Indian J orthop2007, 41: 219 -213

2. Bhabhulkar S, Bhabhulkar S. controversies in the management of intraarticular fractures of distal humerus fracture, Indian J orthopaedics, 2011; 45 : 216-25

3. Gupta R, Khanchandani P. Intercondylar fractures of the distal humerus in adults: A critical analysis of 55 cases. Injury 2002;33:511

4. Muller ME, Schneider R, Willenegger H . Manual of internal fixation. Techniques recommended by AO group. $2^{\text {nd }}$ edition. Springer : new York;1979.p.71 -87

5. Ring D, Gulotta L, Chin K Jupiter JB . Olecronon Osteotomy for exposure of fractures and non unions of the distal humerus. J Orthop Trauma 2004; 18: 446

6. Wilkinson JM, Stanley D. posterior surgical approachs to the elbow: A comparative anatomical study. J Shhoulder Elbow Surgery $2001 ; 10 ; 380-2$

7. Bryan RS, Morrey BF. Extensive posterior exposure of the elbow. Clincal orthopaedics and related research 1982 ; $166: 188-92$
8. Brown RF, Morgan RG. Intercondylar Tshaped fractures of the humerus. Results in ten cases treated by early mobilization. J Bone Joint Surg Br 1971;53:425

9. O'Driscoll SW. The triceps-reflecting anconeus pedicle (TRAP) approach for distal humeral fractures and nonunions. Orthop Clin North Am 2000;31:91-101.

10. Kasser JR, Richards K, Millis M. The triceps-dividing approach to open reduction of complex distal humeral fractures in adolescents: A Cybex evaluation of triceps function and motion. J Pediatr Orthop 1990;10:93-6.

11. Bass RL, Stern PJ. Elbow and forearm anatomy and surgical approaches. Hand Clin 1994;10:343-56.

12. Macko D, Szabo RM. Complications of tension band wiring of olecranon fractures. J Bone Joint Surg Am 1985;67:1396-401.

13. Horne JG, Tanzer TL. Olecranon fractures: A review of 100 cases. J Trauma 1981;21:469-72. 